\title{
VERIFICATION OF THE SENTINEL-4 FOCAL PLANE SUBSYSTEM
}

\author{
C. Williges ${ }^{\mathrm{a} *}$, R. Hohn ${ }^{\mathrm{b}}$, H. Rossmann ${ }^{\mathrm{a}}$, S. Hilbert ${ }^{\mathrm{a}}$, M. Uhlig, K. Buchwinkler ${ }^{\mathrm{a}, \mathrm{b}}$, R. Reulke ${ }^{\mathrm{c}}$, \\ ${ }^{\text {a }}$ Department of Optical Sensor Systems, German Aerospace Center DLR, 12489 Berlin, Germany - \\ (christian.williges, hannes.rossmann, stefan.hilbert, mathias.uhlig, kevin.buchwinkler)@dlr.de \\ b Airbus Defense and Space, 85521 Ottobrunn, Germany - ruediger.hohn@airbus.com \\ ${ }^{\mathrm{c}}$ Department of Computer Science, Humboldt-Universität zu Berlin, Germany - \\ reulke@informatik.hu-berlin.de
}

Commission I, WG I/10

KEY WORDS: Copernicus, Sentinel-4, Radiometry, Earth Observation, Hyperspectral, Verification of Satellite Sensors, Homogeneous Remote Illumination

\begin{abstract}
:
The Sentinel-4 payload is a multi-spectral camera system which is designed to monitor atmospheric conditions over Europe. The German Aerospace Center (DLR) in Berlin, Germany conducted the verification campaign of the Focal Plane Subsystem (FPS) on behalf of Airbus Defense and Space GmbH, Ottobrunn, Germany. The FPS consists, inter alia, of two Focal Plane Assemblies (FPAs), one for the UV-VIS spectral range $(305 \mathrm{~nm} \ldots 500 \mathrm{~nm})$, the second for NIR $(750 \mathrm{~nm} \ldots 775 \mathrm{~nm})$. In this publication, we will present in detail the opto-mechanical laboratory set-up of the verification campaign of the Sentinel-4 Qualification Model (QM) which will also be used for the upcoming Flight Model (FM) verification. The test campaign consists mainly of radiometric tests performed with an integrating sphere as homogenous light source.

The FPAs have mainly to be operated at $215 \mathrm{~K} \pm 5 \mathrm{~K}$, making it necessary to exploit a thermal vacuum chamber (TVC) for the test accomplishment. This publication focuses on the challenge to remotely illuminate both Sentinel-4 detectors as well as a reference detector homogeneously over a distance of approximately $1 \mathrm{~m}$ from outside the TVC. Furthermore selected test analyses and results will be presented, showing that the Sentinel-4 FPS meets specifications.
\end{abstract}

\section{INTRODUCTION}

In this publication we report about the verification campaign of the Sentinel-4 UV-VIS and NIR FPS qualification models, with focus on the opto-mechanical set-up as well as test results. Chapter 2 gives a description of the overall system design and the results of the verification campaign: Sub-chapter 2.1 gives an overview over the Sentinel-4 FPS design, consisting of FPAs and appended electronics. 2.2 deals with the GSE design, the boundary conditions, opto-mechanical solutions, temperature control and automatization approach. 2.3 is about data evaluation and test results. We discuss the gained results and give an outlook for the upcoming FM verification in chapter 3, before we close the publication with acknowledgements and references.

\section{FOCAL PLANE SUBSYSTEM VERIFICATION}

\subsection{Payload Design}

The Sentinel-4 Focal Plane Subsystem (FPS) consists of two FPAs, two Front End Electronics (FEEs) and one Front End Support Electronic (FSE). During verification an Instrument Control Unit (UCI) was also applied as substitute for later on board computation systems. The FPAs house the CCD detectors, the detector-close electronics, as well as internal LEDs for radiometric on-board calibration.

The UV-VIS detector is a split frame detector: designated areas are read out in separate directions (compare Figure 1). This allows for adaptation of integration time and gain settings, depending on wavelengths hitting designated areas. The image zone of UV-VIS I is designed for wavelength range $305 \mathrm{~nm} \ldots 343 \mathrm{~nm}$, UV-VIS II for $343 \mathrm{~nm} \ldots 500 \mathrm{~nm}$. UV-VIS I has the advantage of two different read out directions, with different fixed gain settings, to adapt for different irradiation intensities. For faster read out, UV-VIS II is separated in four single read out registers with same gain settings. Apart from the image zones, it comprises of two storage zones of sizes equal to either UV-VIS I or II, where images will be stored before read out. The storage zones are mechanically shielded from illumination.

The NIR detector comprises of one image area and one storage area of equal size. It has one read out direction and one fixed gain setting.

Each image zone in each detector is covered with a designated anti-reflective coating allowing only designated wavelengths to pass.

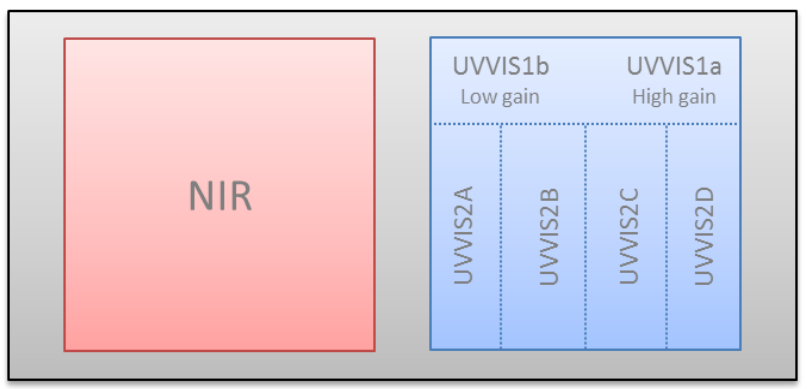

Figure 1: Sentinel-4 detector design of UV-VIS and NIR detector

* Corresponding author 
To maintain excellent operating conditions with low noise the operating temperature of both detectors is $215 \mathrm{~K} \pm 5 \mathrm{~K}$. Temperature transport from the detectors happens via "cold fingers" made from $\mathrm{SiC}$ and a thermal flex harness to the heat pipe. Cooling happens via heat pipes and radiators, while for active temperature control heating elements are attached to the link heat pipe / thermal flex harness.

Apart from both detectors, the FPAs have an operating temperature of approx. $293 \mathrm{~K}$. To guaranty stable operating conditions of the detectors, a thermal insulation is implemented between detectors and FPA (compare Figure 2).

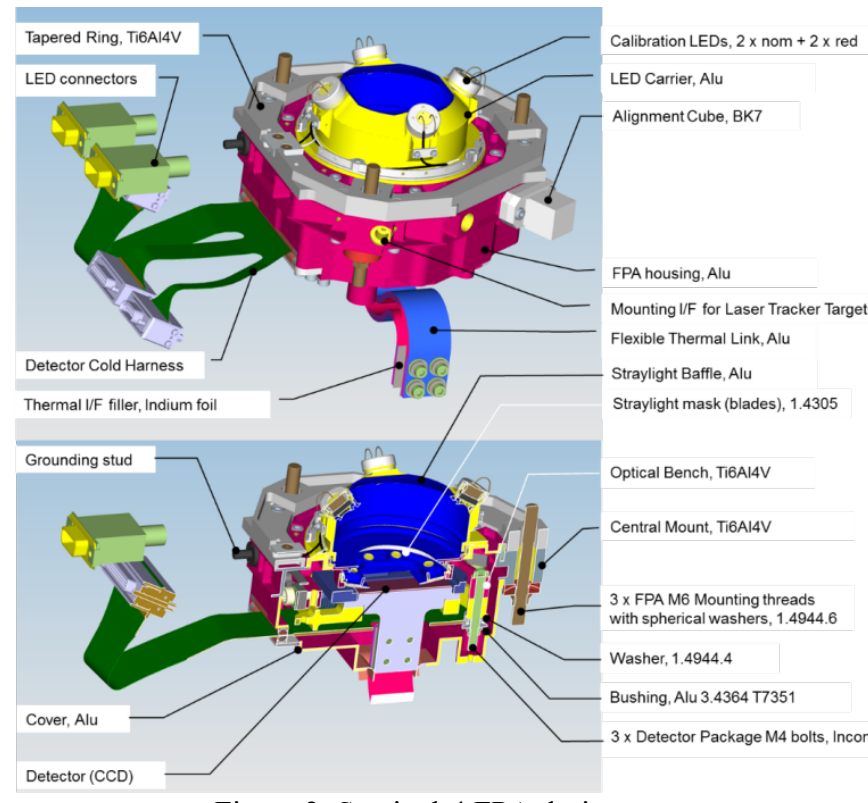

Figure 2: Sentinel-4 FPA design

Each FPA possess an according FEE that converts the analogue signal into digital signals. To minimize losses and noise, FPAs and FEEs are closely aligned and are connected via short electrical flex harness. One FSE provides power supply for FPAs and FEEs and transfers the digital signals to the ICU (compare Figure 3).

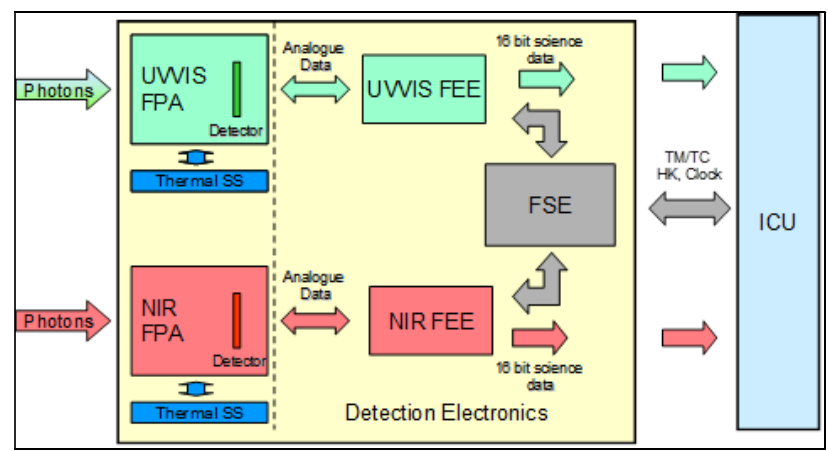

Figure 3: Focal Plane Subsystem Design

\subsection{Design of Test Set-Up}

Verification campaign comprises mostly of radiometric tests. An overview is provided in Table 1. Several tests have been conducted at the very beginning and the end of the verification campaign to test for stability of the FPS equipment.

Radiometric tests have been performed exploiting a light source with high spatial homogeneity and long term stability. An integrating sphere (IS) was used for this purpose, $50 \mathrm{~cm}$ in diameter, aperture $20 \mathrm{~cm}$. For the cross talk test a $635 \mathrm{~nm}$ Diode laser was deployed, the internal LEDs for a remanence test.

The MTF test has been performed on the Engineering Model identical in construction to the QM. Set-up and results will be presented in an additional publication.

\begin{tabular}{|l|c|}
\hline Performed test & Deployed light source \\
\hline Linearity and PRNU & IS, Spectral Filters \\
(Average) Dark Signal & None (Darkness) \\
Memory Effect & Internal LEDs \\
Cross-Talk & 635 nm Diode Laser \\
Stability over 24 h & None (Darkness) \\
Random Telegraph Signal & None (Darkness) \\
FPA LED Calibration & Internal LEDs \\
Quantum Efficiency & IS, Spectral Filters \\
Photo Response Tests & IS, Spectral Filters \\
\hline
\end{tabular}

Table 1: Overview over performed verification tests

2.2.1 Boundary Conditions: The detectors had to be verified under test-as-you-fly conditions: Depending on the specific test the temperature had to be adjusted to $210 \mathrm{~K}, 215 \mathrm{~K}$, $220 \mathrm{~K}$, respectively. The appended electronic (FEEs, FSE) had to be stabilized at $\sim 293 \mathrm{~K}$. Two separated tempering circles have been implemented to control temperature according to requirement.

Additionally operation conditions demanded for vacuum operations to reduce noise and to protect the hygroscopic ARcoating on each detector from degrading caused by humidity. We deployed a thermal vacuum chamber (TVC), able to generate a pressure $<5^{*} 10^{-6} \mathrm{mbar}$ to meet these requirements. Pressure and temperature in our facility are long term stable Prever several weeks.

2.2.2 Opto-Mechanical Test Set-Up: The deployment of integrating spheres usually demands for a positioning of the detector in the light exit aperture (Ducharme, 1997). Our equipment (integrating spheres, filter wheels) is built for application under ambient atmospheric conditions. Additionally, the tight spatial set-up of FPAs and associated FEEs does not allow for placement at the desired position. To overcome these circumstances while simultaneously generating a homogeneous illumination the integrating sphere was placed outside the TVC in a distance, large enough to deploy an outcome of the $\cos ^{4}$-law: For a sufficient large distance between light source and detector and for the correct ratio of size between those two, illumination of an area (e.g. a detector) is homogeneous (Labshere 2016). The IFOV (Instantaneous Field Of View) for all pixels is regarded to be equal in this special case. A schematic of this idea is shown in Figure 4a, the adaption to the verification set-up in Figure $4 \mathrm{~b}$.

The graph in Figure 5 shows the theoretical outcome of the setup calculated via $\cos ^{4}$-law over the diagonal of the UV-VIS detector. Homogeneity of $>99.95 \%$ is theoretically achievable. To manipulate wavelength and intensity, two filter wheels have been integrated into the beam path, one with interference filters the second with neutral density filters (Figure $4 b$ ).

The FPAs with associated electronic boxed will have a certain non-perpendicular and non-parallel orientation on the satellite bus. The flex harnesses linking FPAs to FEEs are tightly adapted to this orientation, with respect to lengths and shapes. The required test-as-you-fly configuration demands for sufficient space in the TVC; we have been constrained to realize a large and complicated mount to meet obligations. 


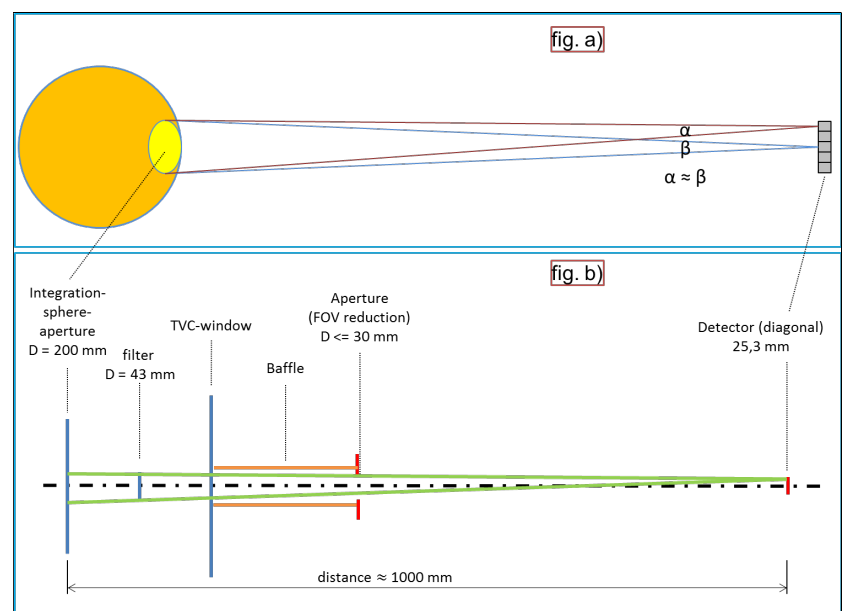

Figure 4: a) principle of homogeneous illumination via remote light source; b) adaption to verification set-up

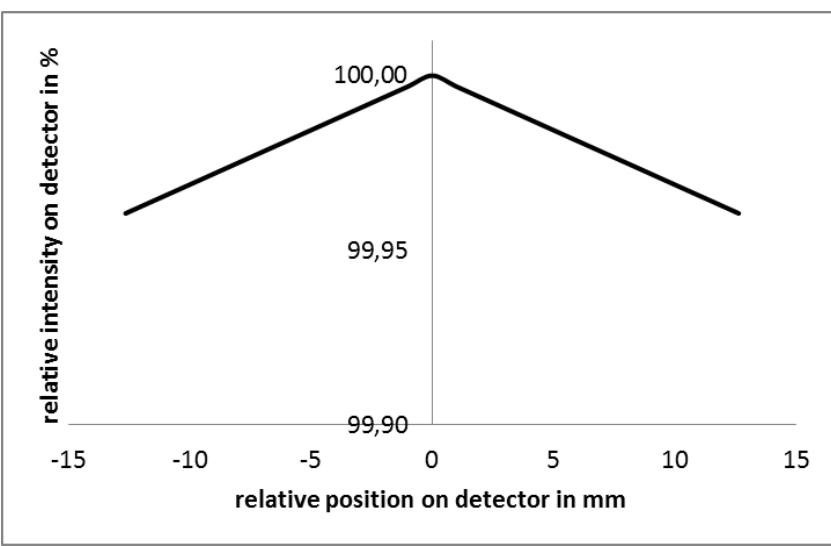

Figure 5: Intensity distribution on the detector from a remote source calculated per $\cos ^{\wedge} 4$ - law. See Figure 4 for dimensions

Due to limited space in front of the TVC's entrance window, we decided for a set-up that mounts the FPAs and a reference detector along and parallel to the optical axis $(\mathrm{OA})$, with the detectors facing the OA perpendicularly. The OA is given by TVC window with attached filter wheels and integrating sphere. A folding mirror on a linear manipulator moving along the optical axis was exploited to subsequently transfer light to each detector. In combination with a spatial off-set of each detector with respect to the $\mathrm{OA}$ this allowed for equal optical path lengths, guarantying equal illumination. A schematic is shown in Figure 6.

For the cross talk test, integrating sphere as well as filter wheels have been removed from set-up and replaced by a laser on a 4-axis manipulator.

2.2.3 Temperature control: As mentioned above, verification of the whole FPS has been performed under operating conditions. Depending on test this has been $210 \mathrm{~K}$, $215 \mathrm{~K}$, and $220 \mathrm{~K}$, respectively, for FPAs and $293 \mathrm{~K}$ for FEEs and FSE. The temperature was long term stabilized to $< \pm 50$ $\mathrm{mK}$.

Two independent temperature controlling circuits have been set-up, one controlling the in series connected FPAs, second one controlling the in series connected electronics. Due to the higher thermal load UV-VIS FPA has been under supply first, NIR FPA second. FEEs and FSE are also in series for the same reasons: UVVIS FEE, NIR FEE, FSE (see Figure 7).

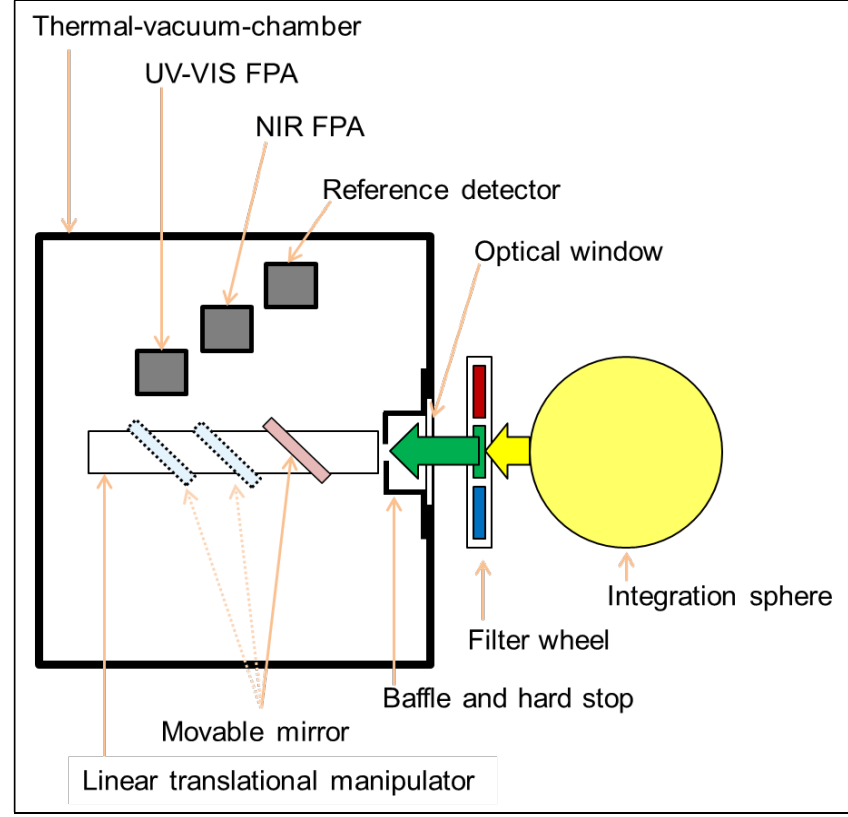

Figure 6: Schematic of test set-up inside and outside TVC

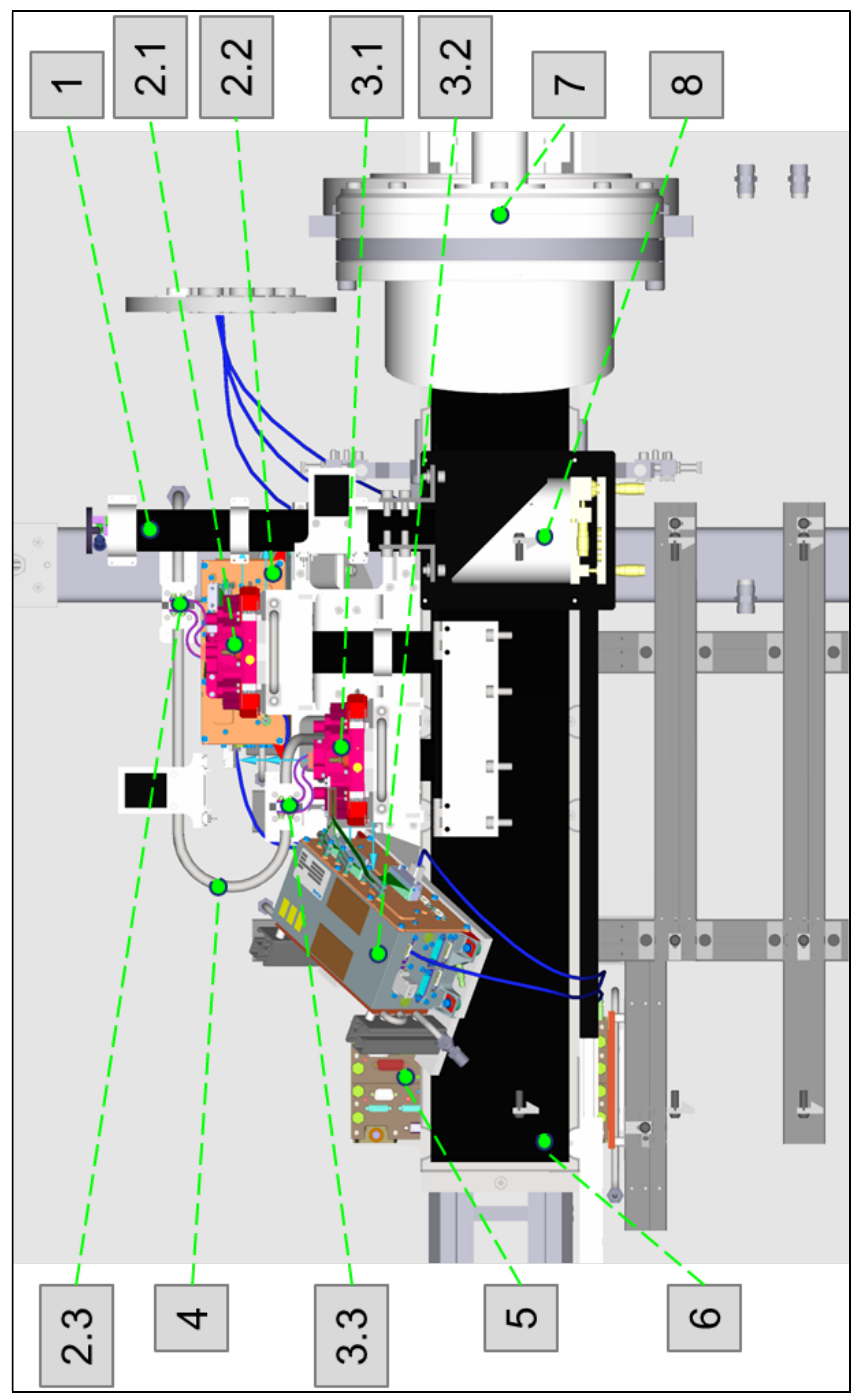

Figure 7: Top view on CAD drawing of internal TVC set-up 


\begin{tabular}{|l|c|}
\hline Label & Labelled item \\
\hline 1 & Reference Detector \\
2 & UVVIS FPA \\
2.2 & UVVIS FEE \\
2.3 & NIR FPA \\
3.1 & NIR FEE \\
3.2 & UVVIS temperature control connection \\
3.3 & NIR temperature control connection \\
4 & FPA temperature circuit \\
5 & FSE \\
6 & Linear manipulator for mirror displacement \\
7 & Optical window of TVC \\
8 & Folding mirror \\
\hline
\end{tabular}

Table 2: Explanation of labelled parts from Figure 7

2.2.4 Automatization of Test Conduction: The amount of tests and repetitions demanded for an automatization process to increase efficiency and reduce possible human failure to a minimum. The automatization software AutoIt ${ }^{\circledR}$ was exploited to control the Matlab ${ }^{\circledR}-$ GUI of the ICU, enabling the automated control of the Sentinel-4-test equipment (e.g. integration times, frame numbers, modes, etc.), as well as control of our GSE (mirror position, temperature, etc.).

\subsection{Data Evaluation and Results}

In this chapter test results of the following verification tests will be presented:

1. Photo Response Non-Uniformity

2. Linearity

3. Non-Linearity

4. System Gain

5. Memory Effect / Remanence

2.3.1 Signal Generation: The expected signal in DN (digital numbers) can be estimated the following equation 1 :

$\hat{\mathrm{S}}[\mathrm{DN}]=\eta_{\mathrm{SG}}^{\mathrm{Det}}\left[\frac{\mathrm{DN}}{\mathrm{e}^{-}}\right] \cdot \eta_{\mathrm{QE}}(\lambda)\left[\frac{\mathrm{e}^{-}}{\mathrm{photon}}\right] \cdot \tau_{\mathrm{int}}[\mathrm{s}] \cdot \mathrm{A}_{\mathrm{Det}}\left[\mathrm{m}^{2}\right] \cdot \frac{\lambda}{\mathrm{hc}}\left[\frac{\mathrm{m}}{\mathrm{Ws}^{2} \mathrm{~m} / \mathrm{s}}\right] \cdot \mathrm{E}\left[\frac{\mathrm{W}}{\mathrm{m}^{2}}\right]+\mathrm{DS}$

where

\begin{tabular}{|l|c|c|}
\hline Symbol & Meaning & Unit \\
\hline$\eta_{\mathrm{QE}}(\lambda)$ & Quantum efficiency & {$\left[\mathrm{e}^{-} /\right.$photon $]$} \\
$\eta_{\mathrm{SG}}^{\text {Det }}$ & Overall system gain & {$\left[\mathrm{DN} / \mathrm{e}^{-}\right]$} \\
$\mathrm{A}_{\mathrm{Det}}$ & Detector area & {$\left[\mathrm{m}^{2}\right]$} \\
$\tau_{\text {int }}$ & Integration time & {$[\mathrm{s}]$} \\
$\mathrm{E}$ & irradiance & {$\left[\mathrm{W} / \mathrm{m}^{2}\right]$} \\
$\mathrm{DS}$ & Temperat. depending dark signal & {$[\mathrm{DN}]$} \\
$\lambda$ & Wavelength & {$[\mathrm{m}]$} \\
\hline
\end{tabular}

Table 3: Symbols, meaning and units mentioned in equation 1

The following noise components are expected to occur: photon noise, dark signal, read noise. As dark and photon noise are Poisson distributed, read noise follows a Gaussian normal distribution. Equation 2 describes the relation:

$$
\sigma_{\mathrm{s}}^{2}=\eta_{\mathrm{SG}}^{2} \cdot \hat{\mathrm{S}}+\sigma_{\mathrm{k}}^{2}
$$

This equation will also be used to describe the overall system gain (Janesick, 2007).

2.3.2 Photo Response Non-Uniformity and linearity: Linearity evaluation has been performed by integration time variation with fixed irradiance. Due to a misaligned aperture, inhomogeneous shading occurred on both FPA detectors, $\mathrm{p}-\mathrm{v}$ approximately $20 \%$ (compare Figure 8a).

The shade had to be estimated and corrected prior to determination of PRNU. Shade correction was performed as following: A linear relation is expected between Signal $\hat{S}_{i j}^{k}$ and a term called hereinafter Exposer (irradiance $\times$ integration time), explained in formula 3 :

$$
\hat{S}_{i j}^{k} \approx a_{i j} \cdot E_{i j}^{k}+b_{i j}
$$

with respect to pixel position (ij) and measurement $\mathrm{k}$ (e.g. by varying integration time). The shade causes a spatial dependency (pixel dependency) of the Exposer-term.

The PRNU correction cannot be handled independently from the shade correction. The correction causes a leveling of each pixel without offset, with respect to a homogeneous reference irradiation $\bar{E}^{k}$, described by a mean increase $c$ given in formula 4 :

$$
c=\operatorname{mean}\left(a_{i j}\right) \quad c_{i j}=\frac{c}{a_{i j}} \quad \tilde{S}_{i j}^{k}=c_{i j} \cdot\left(\hat{S}_{i j}^{k}-b_{i j}\right)
$$

The following analysis will be performed with the corrected signal value $\tilde{S}_{i j}^{k}$, for characterization of PRNU a median based analysis of the slope has been performed (formula 5):

$$
d_{i j}=\operatorname{median}\left(c_{i j}\right) \quad P R N U=\operatorname{stddev}\left(a b s\left(d_{i j}-c_{i j}\right)\right) .
$$

The standard deviation on the maximum dynamic of the measured signal is for PRNU $<1 \%$ for all detectors (requirement has been $<4 \%$ ). Figure 8 shows the shade on the UV-VIS detector and a graphical description of the performed correction.

Figure 9 shows the linearity with and without shading correction. Due to the shading, the different pixels show different saturation maxima.

2.3.3 Non-Linearity and its Correction: The above mentioned approach applies also to the analysis of nonlinearity, by analyzing the maximum deviation from linearity. Figure 10 shows its spatial dependence: Figure 10a shows the spatial distribution of pixels with varying deviation from linearity; Figure 10b shows the non-linearity histogram, where cross-marks indicate the derived histogram, the dotted line a fit onto the derived histogram and the solid line mean value and variance. Obviously, the deviation correlates with the shade; higher signals show a higher deviation (compare Figure 8a with Figure 10a)

The signal derivation of $<80 \mathrm{DN}$ compared to the maximum presentable value of the Sentinel-4 detector of $65,536 \mathrm{DN}$ is $<0.13 \%$. 
Figure 11 shows the slightly curved deviation from linearity for the reference pixels of the UVVIS detector depicted in Figure 9a. Deviations are relatively small (Figure 11a), they can easily be corrected to follow an almost linear behavior (Figure 11b).

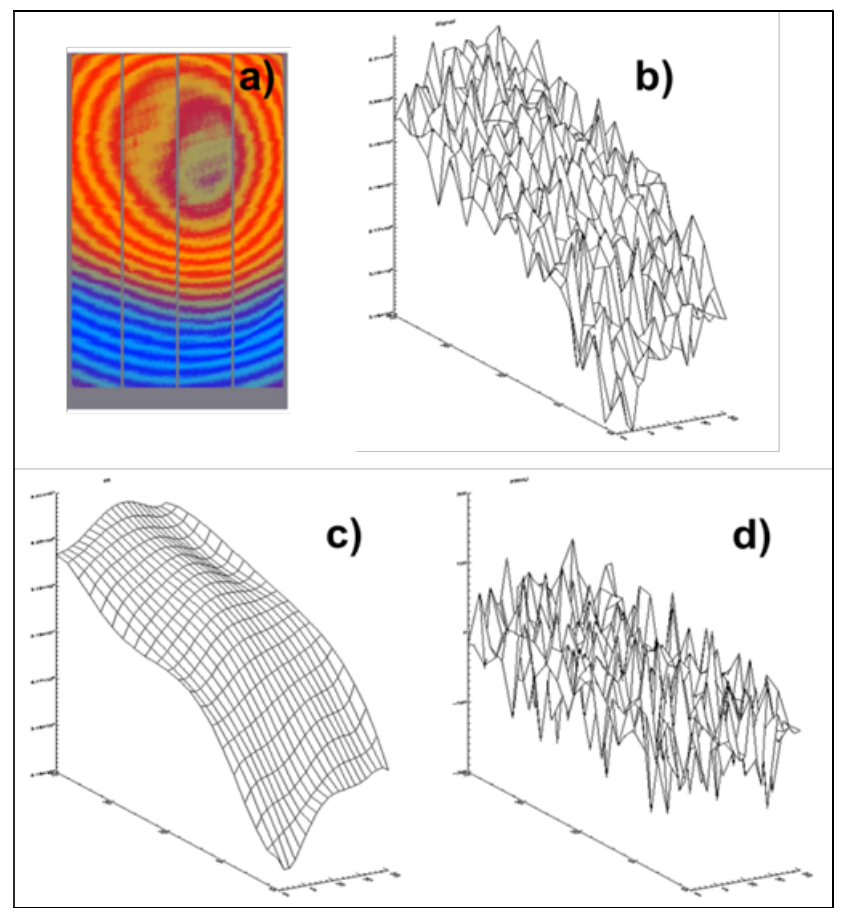

Figure 8: Graphical overview over shading, performed shade correction and PRNU determination. a) inhomogeneous shading on UVVIS detector. Enhanced false color mapping: blue = low signal, red = high signal; b) PRNU and shade combined in one signal; c) calculated shading caused by inhomogeneous illumination; d) corrected signal represents detector PRNU.
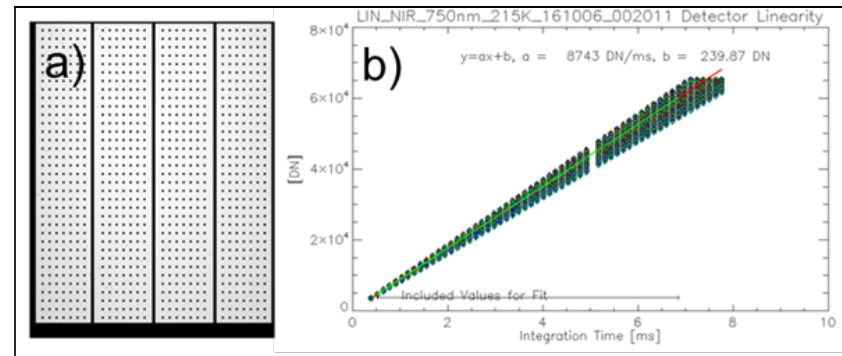

LIN_NIR_750nm_215K_161006_002011 Detector Linearity

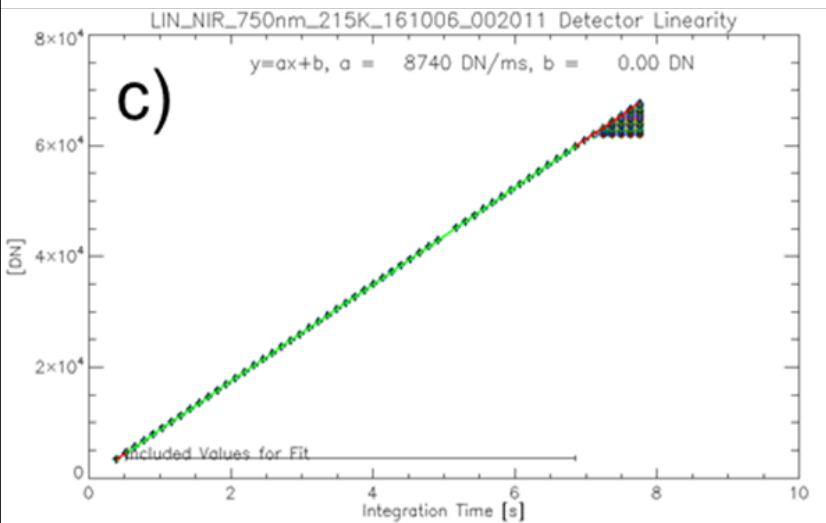

Figure 9: Linearity over UVVIS detector. a) map of analyzed reference pixel of UVVIS detector; b) linearity without shading correction; c) linearity with shading correction.

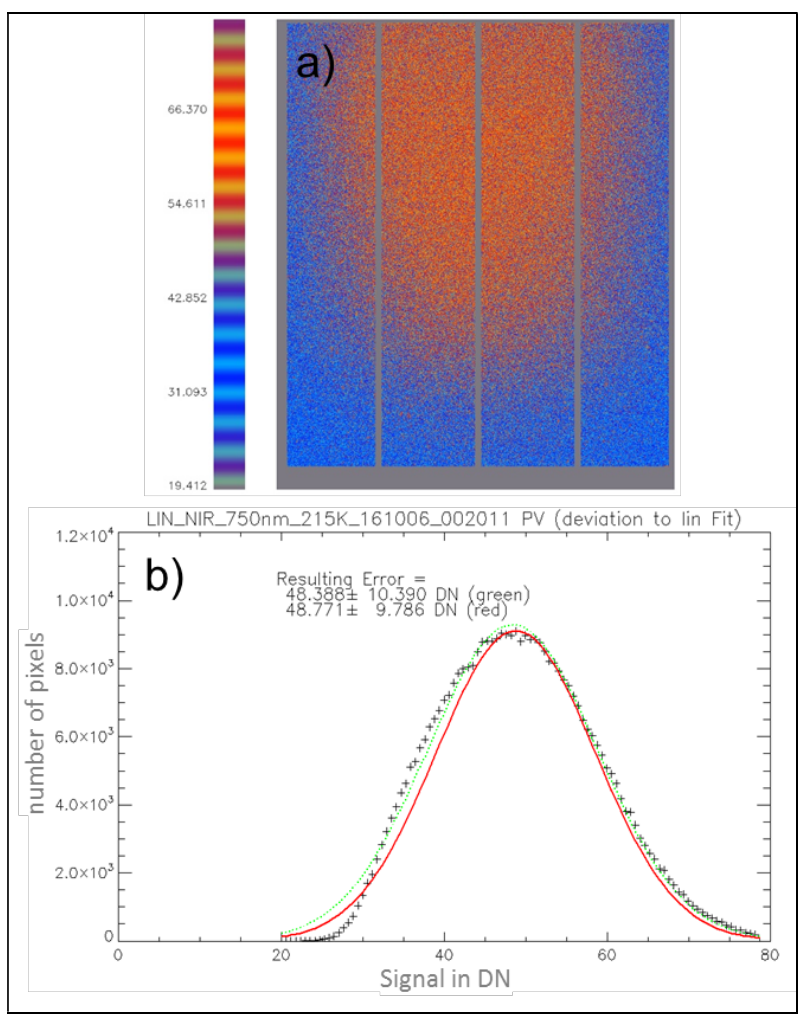

Figure 10: a) mapping of pixels with signal deviation from linearity; b) histogram of number of pixels over signal (deviation) in DN.
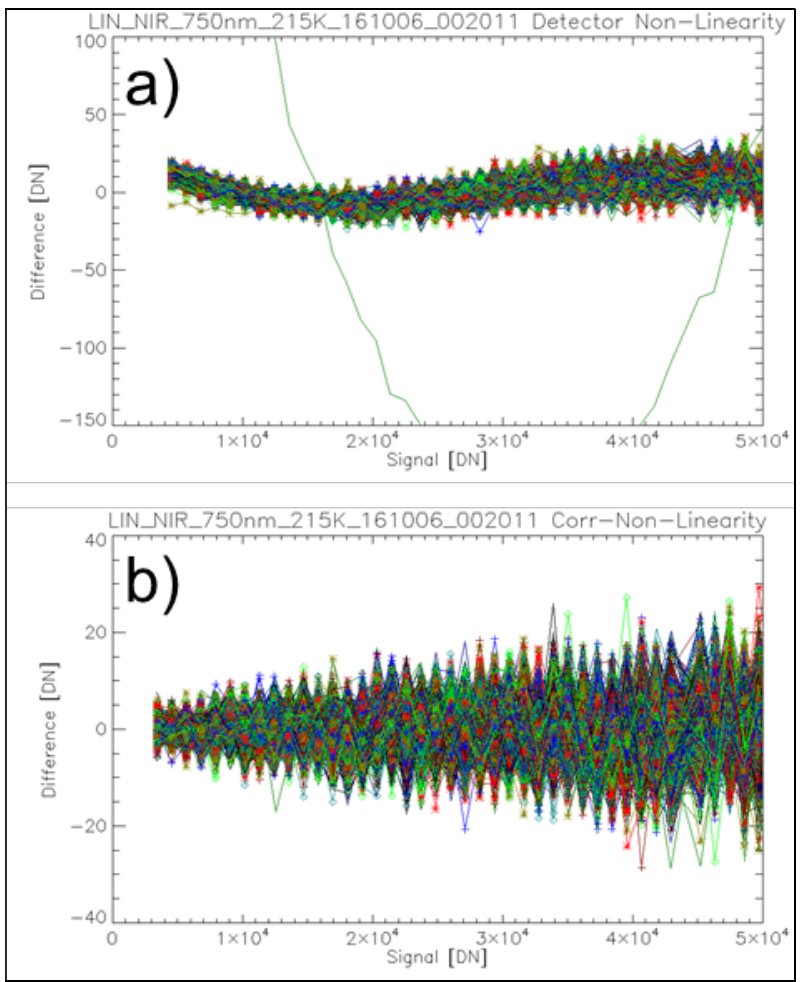

Figure 11: a) uncorrected non-linear behavior of pixels; b) corrected non-linearity. 


\subsubsection{System Gain Determination: Exploitation of system} gain $\eta_{S G}$ allows for translation of generated charge to digital numbers (DN) without knowledge of quantum efficiency, following photon-transfer-method of (Janesick, 2007). The method uses the Poisson-distributed characteristics of incoming radiation and dark current. Figure 12 shows the linear correlation between mean signal and variance of the NIR detector. The relation between charge and $\mathrm{DN}$ is $12 e / 1 \mathrm{DN}$. Figure 13a and Figure 13b depict the spatial dependency, and the histogram for system gain on NIR detector.

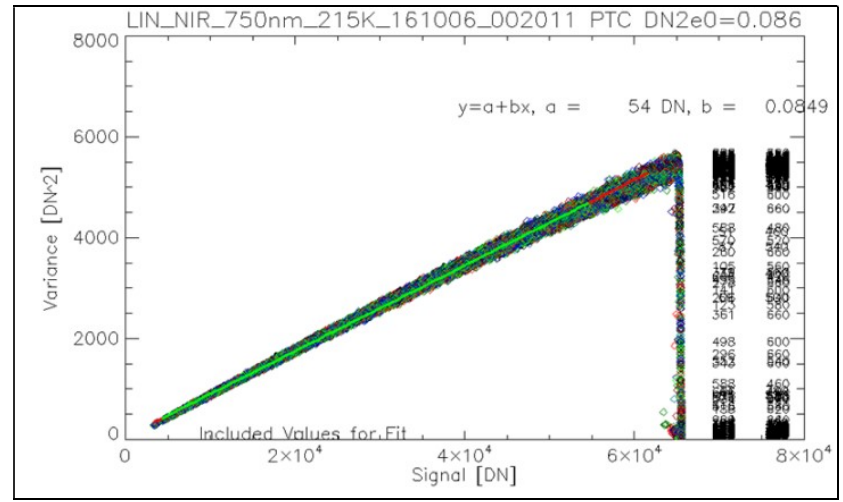

Figure 12: Variance as a function of signal (NIR detector)

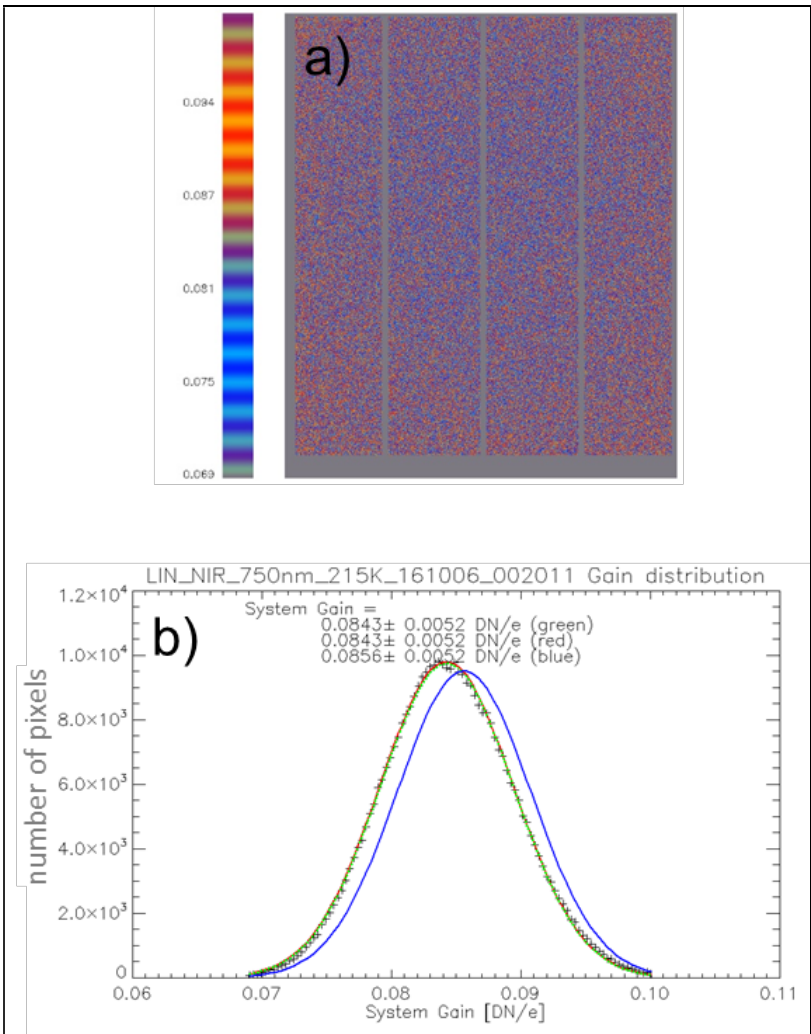

Figure 13: a) spatial dependency of system gain; b) system gain histogram.
2.3.5 Remanence, also known as Residual Bulk Image (RBI), is the effect of remaining charges on exposed areas of the detector, causing "ghost images" on subsequent frames (Janiseck, 2001). For this verification test the FPA-internal LEDs have been exploited. The basic test principle is to intermittently illuminate the detector by named LEDs. They are controlled via external activation mechanism. The basic assumption is that the LEDs can switch off sufficiently fast; fading or glowing does not occur or is in the nano-second (ns) regime.

During image recording with fixed exposure times, an asynchronously pulsed illumination has been triggered externally. Figure 14 depicts exemplary for one pixel of the NIR detector the acquired signal over time.

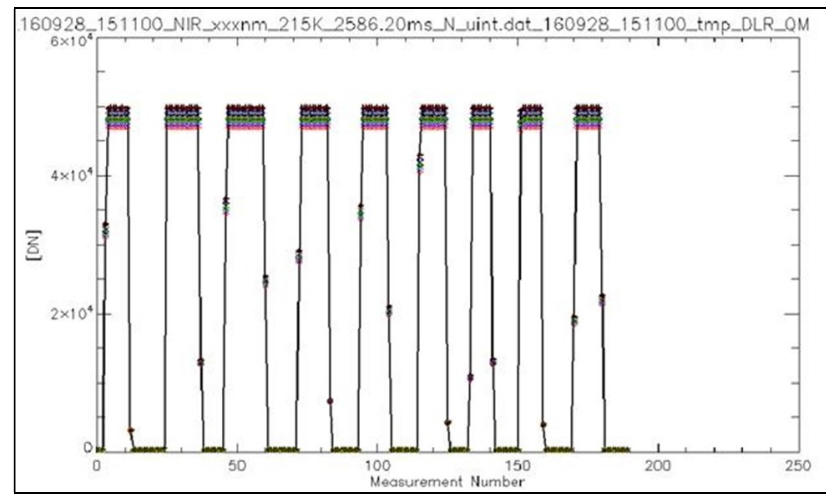

Figure 14: NIR detector output of a sequence of intermittent detector illumination. The image shows the signal of one pixel over time

As can be seen in Figure 14 some of the measurement points are providing neither "bright" nor "dark" information. This indicates that the pixel information has been acquired exactly at that moment where the LEDs for detector illumination have been switched on or off. Here the focus was set on the falling flank i.e. the transit where the LEDs have been switched off.

For the analysis several Pixel have been assessed, located at different positions on the detector. Once the switch off moment has been identified on a measurement number $n$ (image $n$ of the series of images) the average pixel data of the $2 \mathrm{nd}$ and $3 \mathrm{rd}$ following image have been computed to obtain a dark reference. Here the assumption is that the 2nd and 3rd image following the switch off point are not measurably impacted by any remanence effect.

From signal level of the relevant pixel in frame $\mathrm{n}$ this average dark level (of this pixel) has been subtracted to obtain offset corrected information of the "partially illuminated" pixel. In the same way the pixel value of frame $\mathrm{n}+1$ has been offset corrected. Finally both offset corrected results are divided in the manner "partially illuminated" / "first dark". The assumption is that the first dark frame contains any remaining charges in case of existing remanences of the detector and that the amount of charges is proportional to the "previous partially illuminated" frame level why the absolute level of the partly illuminated pixel is mostly irrelevant here.

As the averaged results over all reference pixel of the detector is in the order of $X^{*} 10^{-6}$ (Attenuation in the order of $100 \mathrm{~dB}$ ), we conclude that remanence of the detector is negligible. 


\section{CONCLUSION AND OUTLOOK}

The verification campaign of the Sentinel-4 qualification models of FPAs, FEEs and FSE, has been successfully completed. Our analyzes show that all detectors and appended electronics are within specification. E.g. measured values of PRNU are well below allowed upper limit (Table 4).

\begin{tabular}{|lccc|}
\hline $\begin{array}{l}\text { Measurement Result / } \\
\text { Specification }\end{array}$ & \multicolumn{3}{|c|}{ Detector } \\
\hline & UV-VIS I & UV-VIS II & NIR \\
$\begin{array}{l}\text { Calculated standard } \\
\text { deviation (\%) }\end{array}$ & 0.288 & 0.187 & 0.18 \\
$\begin{array}{l}\text { Calculated amount of } \\
\text { pixel out of 3 } \sigma \text { (in \%) }\end{array}$ & 0.035 & 0.011 & 0.47 \\
$\begin{array}{l}\text { Specified upper limit } \\
\text { (in \%) }\end{array}$ & 4.0 & $\begin{array}{c}2.0 \ldots 1.0 \\
\text { (depending on } \\
\text { wavelength) }\end{array}$ & 4.0 \\
\hline
\end{tabular}

Table 4: PRNU verification: Comparison of measured data to ESA specification

Also non-linearity is very low and can be corrected easily; deviation after correction is $<0.04 \%$.

Considering the obtained results, we are of the opinion that optimization of any part of the FPA-electronics-system for the upcoming flight models is not necessary.

A manual data evaluation of the remanence test showed that the memory effect is smaller than $0.001 \%$. This value gives confidence that the memory values are well within specification.

During verification campaign, an anomaly occurred: the spatial distribution on FPAs should have been homogeneous; instead it showed a shade of $p-v \sim 20 \%$ (compare figure 43a). A misaligned aperture in the beam path has been identified as the root cause of the shading. For the FM verification, any such misalignment will be avoided by adaption of the optomechanical set-up.

\section{ACKNOWLEDGEMENTS}

The presented work has been performed under ESA contract. The authors would like to express their thanks to their respective colleagues at Airbus Defense and Space, ESA and EUMETSAT, and to all partner companies within the SENTINEL 4 industrial consortium for their valuable contributions to the continuing success of this very challenging program. This article has been produced with financial assistance of the EU. The views expressed herein shall not be taken to reflect the official opinion of the EU.

\section{REFERENCES}

Ducharme, A., Daniels, A., Grann, E., \& Boreman, G., 1997. Design of an integrating sphere as a uniform illumination source. IEEE Transactions on education, 40(2), 131-134.

Labshere 2016, Technical Guide - Integrating Sphere Theory and Applications, North Sutton, USA https://www.labsphere.com/site/assets/files/2551/a-guide-tointegrating-sphere-theory-and-applications.pdf (14. April 2017)
Janesick, J. R., 2007. Photon transfer. San Jose: SPIE press.

Janesick, J. R., 2001. Scientific charge-coupled devices (Vol. 83). SPIE press. 